\title{
Development and Performance Evaluation of Steam Channeling Plugging Agent of Thermal Oil Recovery
}

\author{
Wang Chunsheng, Sun Yingfan ${ }^{*}$, He Chenglin, Zhang Haipeng and Du Qiuying
}

\author{
The Northeast Petroleum University, Daqing, Hei Longjiang Province, 163318, China
}

\begin{abstract}
Under the constraints of high temperature and heterogeneity, the common profile control agent can not effectively plug the steam channeling. To address this issue, it is necessary to develop the high temperature resistance steam channeling plugging agent to improve the steam suction profile in the heavy oil reservoir. This paper used Simple Variable Method to optimize the content of the components of the high temperature resistant plugging agent. Static performances evaluation aims to study the influence rules of the formation condition (temperature, salinity and $\mathrm{pH}$ value). Dynamic evaluation is used to study its performance (plugging ratio, residual resistance factor, scouring resistance and thermal stability) in the sand-filled pipe to testify its applicability. The ratio of the component and the injection sequence are shown as follows: $0.03 \%$ coagulant $+2.2 \%$ cross-linking agent $\mathrm{I}+1.2 \%$ cross-linking II $+6 \%$ high efficient main agent. The evaluation experiment results show that the gel can resist at least $280^{\circ} \mathrm{C}$, the plugging ratio is above $93.1 \%$. The plugging ratio only have a $8.43 \%$ reduction after scoured by $15 \mathrm{PV}$ steam $\left(280^{\circ} \mathrm{C}\right)$. After a 10 -day thermal stability experiment $\left(280^{\circ} \mathrm{C}\right)$, the plugging ratio is still above $80 \%$. The result indicated that the plugging agent is suitable for the improvement of steam suction profile. The suggested way of injection is also provided.
\end{abstract}

Keywords: Compound, double fluid method, plugging agent, steam channeling, thermal recovery.

\section{INTRODUCTION}

Currently, high temperature profile control technology mainly consists of the high temperature resistant foam, gel and inorganic particles. Under the precondition of meeting the requirements of heat resistance, foam profile control agent has poor stability, the period of validity of gel profile control agent is short, the particle profile control agent is easy to cause the rigid block and it also has a poor injection performance, and then damage the formation [1]. The mechanical of resins profile control agent is poor, it is easy to plug the formation. For the vertical permeability differences, we should use gel plugging agent with low deformation and liquidity under the precondition of meeting the requirements of heat resistance, it has effective adjustment block effect [2-5].

This project adopts the modification technology to improve the heat-resistant ability of the traditional high temperature resistant main agent, with the coagulant and two types of cross-linking agent, they form a space mesh quadripolymer gel and then recombine with new type of high temperature resistance inorganic particle; Through the perfect evaluation system to systematically describe the sensitivity, adaptability and effectiveness of the blocking agent to the formation environment. It is an ideal profile control agent. This study provides a reference for other hightemperature profile control technology research and has a guiding significance for site construction applications.

\footnotetext{
*Address correspondence to this author at the Northeast Petroleum University, Daqing, Hei Longjiang Province, 163318, China; Tel: 15845985515; E-mail: 939191700@qq.com
}

\section{THE DEVELOPMENT OF PLUGGING AGENT SYSTEM}

$65 \%$ concentrated nitric acid is used in experiments by a certain percentage which makes a modification reaction with the traditional high-temperature main agent on the basis of the original molecules, increasing nitro to improve heat resistance performance. By contrast colloidal temperature resistance performance, we can optimize the best component contents of the main agent.

High temperature resistant test results show that when the solid-liquid ratio is $1: 6$, it has the least amount of dehydration at high temperatures after the main generated agent is gelled, and it stills keep high strength and toughness. The $\mathrm{pH}$ of the preparation of modified high temperature resistant main agent product is between $2 \sim 3$ in the end, the $\mathrm{pH}$ of aqueous solution value is neutral, when the mass fraction of main agent is greater than or equal to $14 \%$, the solution is saturated and becomes dark red. The introduction of the nitro makes the stability of benzene of the main agent stronger enough to resist high temperature. Under the environment, the substitution reaction, condensation reaction and crosslinking reaction occur between the molecules. Eventually, the reactants generate a kind of semisolid quaternary copolymer gel colloid with pyknotic space mesh structure, which is the main reason of its high temperature resistance [6-9].

\section{RATIO OPTIMIZATION EXPERIMENTS}

The experiments aim to optimize the content of each component, we use the gelling properties (gelling time, gelling viscosity) as the screening criteria. The temperature of 
simulated formation is $200^{\circ} \mathrm{C}$, the $\mathrm{pH}$ of the plugging agent solution is 7 .

Experiment drugs: Coagulant, Modified main agent, cross-linking agent I, cross-linking agent II, $\mathrm{pH}$ adjusting agent, formation water.

Experiment instruments: Hydrothermal synthesis reactor, electric balance, Brookfield rotary viscometer, incubator, electric blender, $\mathrm{pH}$ meter, measuring cylinder, beaker and so on.

Experiment method: Weighing a certain quality of the modified-main agent, cross-linking agent I and coagulant, sufficiently dissolved in the water, respectively, adjusting the $\mathrm{pH}$ value to 7 . Mixing the three kinds of solutions, then adding the cross-linking agent II into mixed solution. The mixed solution is divided into ten parts approximately, putting each part into hydrothermal synthesis reactors, sealed, placed in the reaction environment $\left(200^{\circ} \mathrm{C}\right)$ taking out one reactor every one hour, it should be cooled to room temperature in the air, then opened the reactor (the reactor cannot be opened in the high temperature), should be used the routine method to measure the viscosity of gel in the reactor with viscometer, select the appropriate rotor and rotate, when the number fluctuated in a range with a small amplitude, average sould be recorded. The ratio of each component in the solution using the above procedure [10-15] should be changed.

\subsection{The Influence of HPAM on the Plugging System}

The contents are shown as follows: HPAM + crosslinking agent $\mathrm{I}+$ cross-linking agent $\mathrm{II}=+0.03$ wt. $\%+2.2$ wt. $\%+1.5$ wt. $\%$. We will investigate the effects of the content of HPAM according to the gelling properties of the plugging agent system.

As shown in Fig. (1), the viscosity increases when the content of HPAM increases, the gelling time reduces. It has a role in promoting the formation of the gel. The content of HPAM is in direct proportion to the initial viscosity of the solution, we can change the content to make it easier to inject. After an overall consideration, the content of HPAM is 0.03 wt. $\%$.

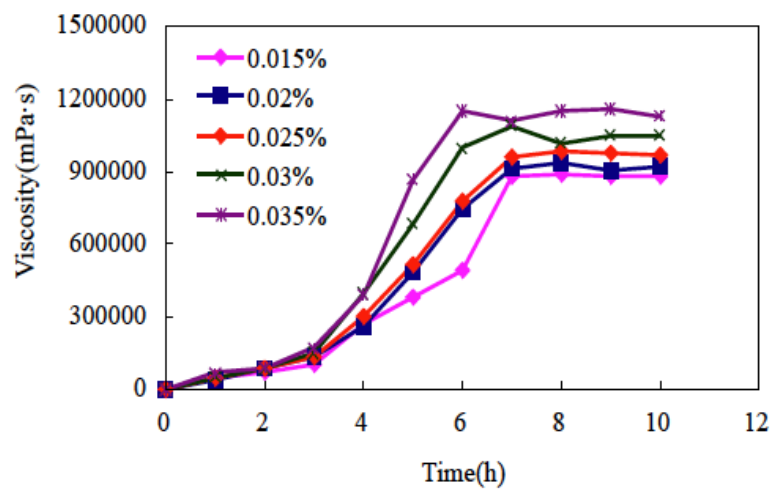

Fig. (1). The influence of HPAM on the gel system.

\subsection{The Influence of the Modified High Temperature Resistant Main Agent on the Plugging Agent System}

The contents are shown as follows: HPAM + crosslinking agent $\mathrm{I}+$ cross-linking agent $\mathrm{II}=0.03$ wt. $\%+2.2$ wt. $\%+1.5$ wt. $\%$. We will study the effects of the content of main agent according to the gelling properties of the plugging agent system.

As shown in Fig. (2), the content of the main agent is in direct proportion to the gelling viscosity, but inversely proportional to the gelling time. When the content of the main agent is $6 \%$, the gelling time is the longest, the content of the main agent is between $6 \%$ and $10 \%$, the gelling viscosity changes a little. As the main agent, the phenolic hydroxy in the molecule can connect with the cross-linking agent through covalent bond, the suitable content contributes to the denser structure and better high temperature resistance. After an overall consideration, the content of the main agent is $6 \%$.

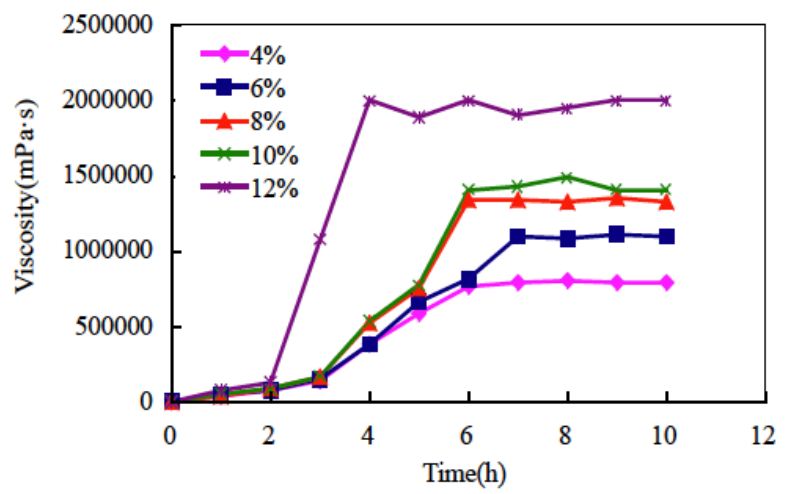

Fig. (2). The influence of the content of main agent on the gelling time and the gelling viscosity.

\subsection{The Influence of the Cross-linking Agent I on the Gel System}

The contents are shown as follows: Main agent + HPAM + cross-linking agent II $=6 \mathrm{wt} . \%+0.03 \mathrm{wt} . \%+1.5 \mathrm{wt} . \%$. We will investigate the effects of the content of cross-linking agent I according to the gelling properties of the plugging agent system.

When the content is $2.2 \%$, the mole ratio of cross-linking agent I and cross-linking agent II is 2:5. Intermolecular cross-linking degree is the largest, when the content of crosslinking agent increases, the viscosity decreases; The gelling time reduces when the content of cross-linking agent I increases Fig. (3). generally, the content of cross-linking agent $\mathrm{I}$ is $2.2 \%$.

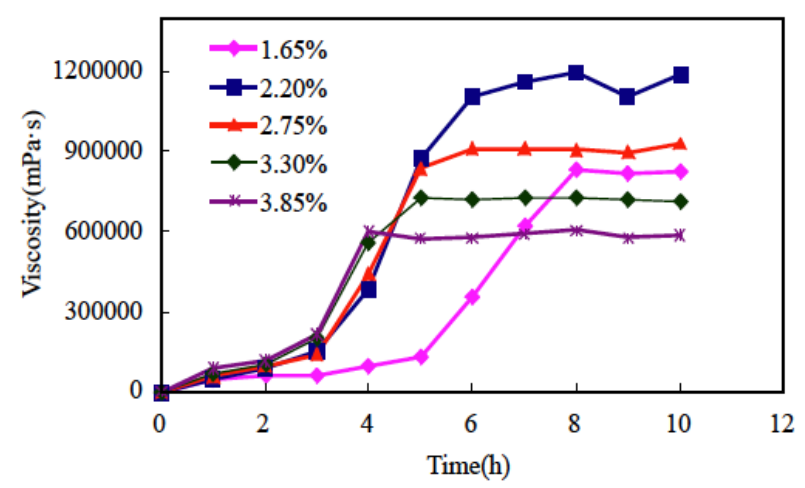

Fig. (3). The influence of the content of cross-linking agent I on the gelling time and the gelling viscosity. 


\subsection{The Influence of the Cross-linking Agent II on the Plugging Agent System}

The contents are shown as follows: Main agent + HPAM + cross-linking agent I $=6 \mathrm{wt} . \%+0.03$ wt. $\%+2.2$ wt. $\%$. We will investigate the effects of the content of cross-linking agent II on the gelling properties of the plugging agent system.

The content of cross-linking agent II is in direct proportion to the gelling viscosity, but inversely proportional to the gelling time. When the content of cross-linking agent II is more than $1.2 \%$, the final gel is in a semi-solid state, the gel strength and the gelling time is less than 4 hours, which show that the gelling time is short Fig. (4).

At last, the formula and the suitable ratio of components are shown as follows: $0.03 \%$ coagulant $+2.2 \%$ cross-linking agent I $+1.2 \%$ cross-linking II $+6 \%$ highly efficient main agent.

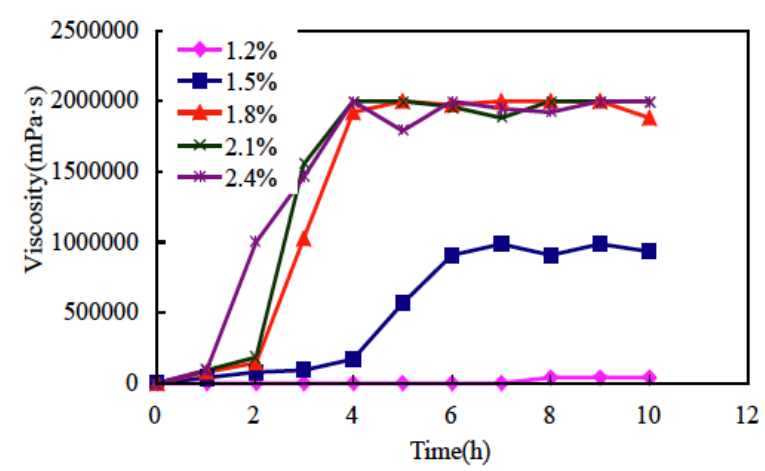

Fig. (4). The influence of the content of cross-linking agent II on the gelling properties.

\section{STATIC PERFORMANCE EVALUATION OF PLUGGING AGENT SYSTEM}

\subsection{The Influence of Temperature on the Gel Properties}

The reaction temperatures of the compare experiment are $200^{\circ} \mathrm{C}, 240^{\circ} \mathrm{C}, 280^{\circ} \mathrm{C}$, we will examine the impact of temperature on the gel properties (Table 1).

Experimental results show that when the reaction temperature is $240^{\circ} \mathrm{C}$, the gelling viscosity of the plugging agent is up to $65624 \mathrm{mpa} \cdot \mathrm{s}$. High temperature viscosity is between $10000 \mathrm{mpa} \cdot \mathrm{s}$ and $60000 \mathrm{mpa} \cdot \mathrm{s}$. The agent system has the advantage of high efficiency and the wide range of gelling temperature.

Table 1. The influence of temperature on gelling time and viscosity.

\begin{tabular}{|c|c|c|c|}
\hline $\mathbf{T} /{ }^{\circ} \mathbf{C}$ & $\mathbf{2 0 0}$ & $\mathbf{2 4 0}$ & $\mathbf{2 8 0}$ \\
\hline \hline gelling viscosity $/ \mathrm{mPa} \cdot \mathrm{s}$ & 37592 & 64750 & 13797 \\
\hline gelling time $/ \mathrm{h}$ & 8 & 7 & 5 \\
\hline
\end{tabular}

\subsection{The Influence of Salinity on Gel Properties}

Experimental results show that when the salinity is between $1600 \mathrm{mg} / \mathrm{L} \sim 3200 \mathrm{mg} / \mathrm{L}$, the viscosity of the plugging agent changes a little, and gelling time increases. When salinity is more than $4800 \mathrm{mg} / \mathrm{L}$, it cannot be gelled or remain in a low viscosity level.

\subsection{The Influence of pH Value on Gel Properties}

Reaction temperature is $200^{\circ} \mathrm{C}$, we will study the effects of $\mathrm{pH}$ on gel properties. Experiments show that when $\mathrm{pH}$ is between $6 \sim 7$, it has a little influence on gelling viscosity and gelling time, the viscosity of the system decreases, as the gelling time increase. When the $\mathrm{pH}>9$, the change of the gelling viscosity is irregular over time, it cannot gel.

\section{DYNAMIC EVALUATION TEST}

The devices of experiment and the ways of their connection are shown in Fig. (5).

Experiment method:

(1) Preparation of the sand-filled tube: Turn the sand-filled tube into vacuum with the vacuum pump, water-saturated it after half an hour. Then, measure the pore volume and the permeability before plugging.

(2) The seal test: connecting the experiment devices according to Fig. (6). Turn on the valves $\mathrm{c}$ and $\mathrm{d}$, turn off the valve $a$ and $b$. Injecting water through the pump to find the place that is not sealed. Change or tight the connection until it is well sealed.

(3) Plugging experiment: Filled the profile agent solution in the pistons container. Turn on the valves $a, b, d$, turn off the valve c. Displace 3PV profile control agent into sand filling tube at a constant speed of $1 \mathrm{ml} / \mathrm{min}$, keep it sealed, and put it into the thermostat at the temperature of $200^{\circ} \mathrm{C}$ for 12 hours until it is gelled.

(4) The different dynamic evaluation tests are done in following steps.

\subsection{Plugging Ratio, Residual Resistance Factor and Scouring Resistance}

(1) Plugging ratio and residual resistance factor:

After the profile agent gelled, measure the water phase permeability in the air condition. Injecting water at the speed of $1 \mathrm{ml} / \mathrm{min}, 3 \mathrm{ml} / \mathrm{min}, 5 \mathrm{ml} / \mathrm{min}, 7 \mathrm{ml} / \mathrm{min}, 9 \mathrm{ml} / \mathrm{min}$, calculate the water phase permeability, respectively, then get the average. The plugging ratio and residual resistance factor are calculated by formula (1) and formula (2).

$$
\begin{aligned}
& \text { plugging ratio: } E=\left(K_{l}-K_{2}\right) / K_{l} \times 100 \% \\
& \text { Residual Resistance Factor: } R R F=K_{l} / K_{2}
\end{aligned}
$$

Where $K_{1}$ is the water phase permeability before plugging, $K_{2}$ is the water phase permeability after plugging.

Comparing the plugging ratio and residual resistance factor of gel system and composite system at different temperatures $[16,17]$.

\section{(2) Scouring resistance}

Injecting 30PV steam into the sand filling tube at the rate of $3 \mathrm{ml} / \mathrm{min}$ after being sealed, Simulation environment temperature is $280^{\circ} \mathrm{C}$, the back pressure is $2.4 \mathrm{MPa}$. Measuring 


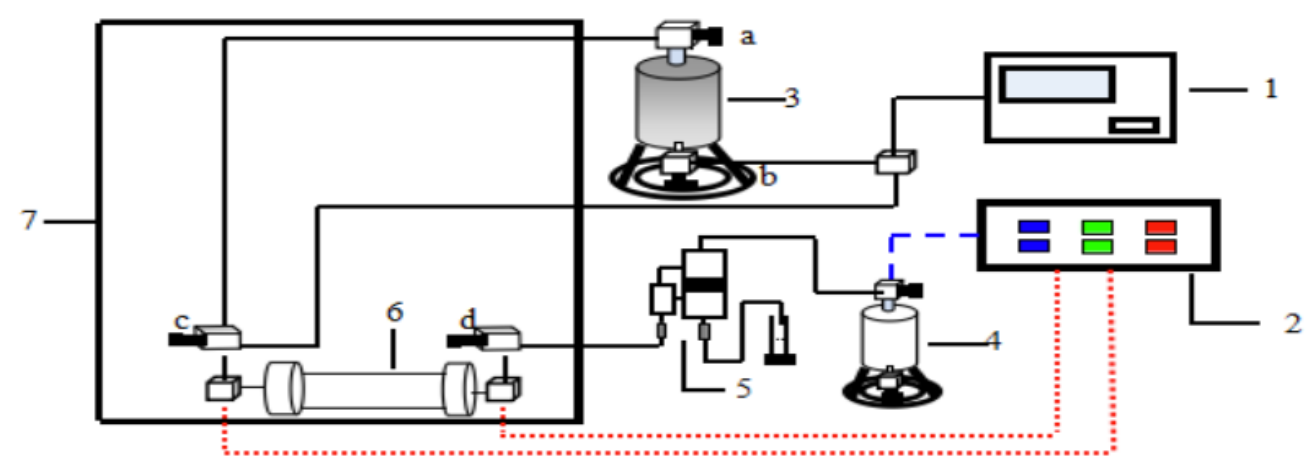

1. Constant flow pump, 2. Pressure monitoring device, 3. The pistons container,

4. Steel pressure vessels, 5. Back-pressure valve, 6. Sand-filled tube; 7. Thermotank $\left(\max : 300^{\circ} \mathrm{C}\right)$.

Fig. (5). The experimental flow chart.

Table 2. Result of plugging ratio and residual resistance factor.

\begin{tabular}{|c|c|c|c|c|c|}
\hline \multirow{2}{*}{ Type } & \multirow{2}{*}{ T( $\left.{ }^{\circ} \mathbf{C}\right)$} & Perm-plug method (md) & \multicolumn{2}{|c|}{ Water permeability measurement (md) } & \multirow{2}{*}{ Plugging ratio (\%) } \\
\cline { 4 - 7 } & & & Before plugging & After plugging & \\
\hline \hline \multirow{3}{*}{ Gel } & 200 & 3538 & 712 & 20 & 97.19 \\
\cline { 2 - 7 } & 240 & 2830 & 675 & 14 & 97.93 \\
\cline { 2 - 7 } & 280 & 3476 & 667 & 46 & 93.6 \\
\hline
\end{tabular}

Table 3. The result of scouring test.

\begin{tabular}{|c|c|c|c|c|c|c|c|}
\hline \multirow{2}{*}{$\begin{array}{c}\text { perm-plug method } \\
\text { (md) }\end{array}$} & \multicolumn{3}{|c|}{ Water permeability measurement (md) } & \multicolumn{3}{c|}{ plugging ratio (\%) } \\
\cline { 2 - 8 } & before plugging & After plugging & $\mathbf{1 5 P V}$ & $\mathbf{3 0 P V}$ & After plugging & $\mathbf{1 5 P V}$ & $\mathbf{3 0 P V}$ \\
\hline \hline 3538 & 712 & 20 & 80 & 355 & 97.19 & 88.76 & 50.14 \\
\hline
\end{tabular}

the water phase permeability after scoured, compares the plugging ratio before and after scoured.

As shown in Table 2, plugging rate achieves at least 93\% under different reaction temperatures. Plugging effect is slightly changed with high temperature. As a consequence, the profile agent has a perfect performance of plugging.

Table 3 shows that plugging ratio is still above $83 \%$ after $15 \mathrm{PV}$ steam washed. When it increases to $30 \mathrm{PV}$, permeability becomes $50.14 \%$. Permeability declines to half it is still enough to adjust the formation permeability differences; it still reflects the characteristics that plugging agent can be degraded and broke (Table 3 ).

\subsection{Thermal Stability}

Under the condition of high temperature, polymers are prone to degradation to hydration. In the steam flooding, retention ability of a profile control agent can be measured by thermal stability through the curve of time-plugging ratio. The simulation of formation temperature is $280^{\circ} \mathrm{C}$.

At a temperature of $280^{\circ} \mathrm{C}$, plugging ratio is still above $80 \%$, after 10 days, the thermal stability curve gradually becomes steady. The plugging profile control agent is slightly degraded under high temperature Fig. (5).

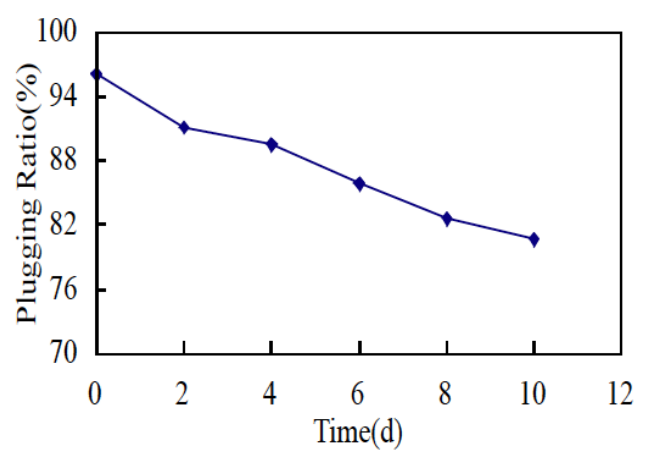

Fig. (6). Result of thermal stability.

\section{CONSTRUCTION PROCESS PARAMETERS}

(1) Profile control agent

Calculate profile agent dosage according to Plugging radius:

$$
V=\pi R^{2} H \phi
$$

Where $V$ is dose of profile control agent, $\mathrm{H}$ is processing layer thickness, $\mathrm{R}$ is average of process radius, and $\Phi$ is porosity. In addition, we should consider the scene construction wastage. 


\section{(2) Injection pressure}

The injection pressure of the profile control agent should be higher than the start-up pressure of high permeable zone. It should be lowered than the start-up pressure of low permeability layer to ensure that the majority of profile control agent prioritize into high permeable formation [18,19]. According to the actual situation of the formation to determine the reasonable injection pressure is:

$$
0.8 P_{B} \geq P_{\mathrm{I}} \geq P_{\mathrm{S}}+P_{\mathrm{fr}}+P_{H}
$$

Where $P_{B}$ is processing layer fracture pressure, $M P a ; P_{I}$ is the injection pressure of oil displacement agent, $\mathrm{MPa} ; \mathrm{P}_{\mathrm{F}}$ is processing layer formation pressure, $\mathrm{MPa} ; \mathrm{P}_{\mathrm{fr}}$ is friction loss during the injection oil displacement agent, $\mathrm{MPa} ; \mathrm{P}_{\mathrm{H}}$ is the well fluid column pressure, MPa.

\section{(3) Injection speed}

Adopting the method of two-fluid process at a low pressure and low emissions, the injection speed ranges from $0.2 \mathrm{~m}^{3} / \mathrm{min}$ to $0.4 \mathrm{~m}^{3} / \mathrm{min}$.

\section{(4) Replace liquid dosage}

After the construction of profile control, in the near well bore zone, in order to not to make high temperature profile control agent to cause congestion, prevent the steam around and pressure-out and avoiding to dilute the gel plugging agent. Generally, the dosage to replace liquid is $15 \%$ of the entire displacement profile control agent [16-20].

\section{CONCLUSION}

(1) The main agent after being modified makes the gel withstand with high temperature at least $280^{\circ} \mathrm{C}$. The results of optimization experiment indicated that HPAM is used to promote the formation of the gel, the main agent is the critical reason of the high temperature resistant performance, the two kinds of cross-link provide the close connection between molecules. Finally, the suitable contents of components are shown as follows: $0.03 \%$ coagulant $+2.2 \%$ cross-linking agent $\mathrm{I}+1.2 \%$ cross-linking II $+6 \%$ high efficient main agent.

(2) Evaluation experiments show that plugging agent has low initial viscosity, applicable to the $\mathrm{pH}=6 \sim 8$, it has a large range of reaction temperature (from $200^{\circ} \mathrm{C}$ to $280^{\circ} \mathrm{C}$ ) and a good salt resistance, plugging ratio is above $93.1 \%$, it has an excellent performance of thermal stability and souring resistance. The result shows that the plugging agent is easy to inject and has a good effect on profile control.

(3) The plugging agent injected in the liquid form with the method of double-fluid under the condition of low pressure and low emissions during the inject operation. Due to the heterogeneity of the formation, liquids are easier to access into the high permeability layer preferentially. Under the environment of formation, the gel plugs the steam channel and forces the steam to change to the low permeability layer. The steam channeling plugging agent is suitable for the improvement of steam suction profile.

\section{CONFLICT OF INTEREST}

The authors confirm that this article content has no conflict of interest.

\section{ACKNOWLEDGEMENTS}

Thanks to the equipment support from the unit and the effort of experimenters, they conquer research difficulties step by step.

\section{REFERENCES}

[1] Q. H. Liu, H. H. Pei, Y. Wang, P. Jiang, Z. W. Wang, and H. T. Li, "Research progress of heat-resistant profile control agent", Oilfield Chemistry, vol. 1, pp. 36, 2013.

[2] S. Tong, and Y. J. Liu, "The application and prospect of profile control and plugging agent with high temperature resistant in steam thermal recovery", Sino-Global Energy, vol. 17, no. 1, pp. 47-50, 2012.

[3] C. H. Song, C. X. Zhao, H. X. Wu, X. W. Han, Y. J. Liu, and J. J. Han, "Technological research on BQ 10block steam drive profile control channeling block", Petroleum Geology and Engineering, vol. 1, pp. 101-103, 2010.

[4] Y. Z. Duan, "Study on multisystem combined with chemical profile control and displacement for heavy oil thermal recovery", Doctoral dissertation, China University of Petroleum, 2010.

[5] W. X. Zhang, "Comprehensive regulation technique research and application of stream flooding in Qi 40 Block", Master's thesis, Northeast Petroleum University, 2013.

[6] X. Zhang, "Research on technologies and its mechanism for improving efficiency of steam flooding in medium depth heavy oil reservoir", Doctoral dissertation, Northeast Petroleum University, 2011.

[7] R. L. Eson, and R. W. Cooke, “A successful high-temperature gel system to reduce steam channeling”, In: SPE Annual Technical Conference and Exhibition, Society of Petroleum Engineers, 1992.

[8] B. L. Hunter, R. S. Buell, and T. A. Abate, "Application of a polymer gel system to control steam breakthrough and channeling", In: SPE Western Regional Meeting. Society of Petroleum Engineers, 1992.

[9] C. Daia, Q. Youb, L. Hec, and F. Zhaoa, "Study and field application of a profile control agent in a high temperature and high salinity reservoir", Energy Sources, Part A: Recovery, Utilization, and Environmental Effects, vol. 34, no. 1, pp. 53-63, 2011.

[10] X. Tang, Y. Liu, H. Qin, L. Cai, and R. Jiang, "A new method of in-depth profile modification for high-temperature and high-salinity reservoir", In: SPE Asia Pacific Oil and Gas Conference and Exhibition. Society of Petroleum Engineers, 2004.

[11] P. W. Chang, I. M. Goldman, and K. J. Stingley, "Laboratory studies and field evaluation of a new gelant for high-temperature profile modification", In: SPE Annual Technical Conference and Exhibition. Society of Petroleum Engineers, 1985.

[12] Q. You, Y. Tang, C. Dai, P. Shuler, Z. Lu, and F. Zhao, "Research on a new profile control agent: dispersed particle gel", In: SPE Enhanced Oil Recovery Conference. Society of Petroleum Engineers, 2011.

[13] S. Chen, "Profile control technology of thermal recovery in Shengli oilfield", In: Asia Pacific Oil and Gas Conference, 1998, pp. 387392.

[14] H. Zhao, P. Zhao, Q. Wang, H. Chen, and S. Zhang, "Using gel prepared by associating polymer to control conformance in high temperature and high salinity reservoirs", In: Canadian International Petroleum Conference. Petroleum Society of Canada, 2004.

[15] C. Wang, H. Liu, J. Wang, C. Hong, X. Dong, Q. Meng, and Y. Liu, "A novel high-temperature gel to control the steam channeling in heavy oil reservoir", In: SPE Heavy Oil Conference-Canada. Society of Petroleum Engineers, 2014.

[16] S. Bauer, P. Gronewald, J. Hamilton, and A. Mansure, "Hightemperature plug formation with silicates", In: Proceedings $30^{\text {th }}$ Workshop of Geothermal Reservoir Engineering. 2005.

[17] R. D. Hutchins, H. T. Dovan, and B. B. Sandiford, "Field applications of high temperature organic gels for water control", In: Symposium on Improved Oil Recovery, 1996, pp. 419-426. 
[18] L. N. Morgenthaler, and H. A. Schultz, "A novel process for profile control in thermal recovery projects", In: SPE Annual Technical Conference and Exhibition. Society of Petroleum Engineers, 1994.

[19] Q. H. Feng, S. Wang, P. Liu, P. Ge, S. Shi, and Y. Sun, "Numerical simulation for the profile control combination process of steam foam and stiff particles", In: SPE Heavy Oil Conference Canada. Society of Petroleum Engineers, 2012.

[20] M. A. Samir, W. Hassan, S. Kamal, A. Hassan, M. Draz, and S. A. Waheed, "Evaluating steam injection profile with high temperature memory PLT", In: North Africa Technical Conference and Exhibition. Society of Petroleum Engineers, 2010.

Received: March 26, 2014

Revised: November 19, 2014

Accepted: January 01, 2015

(c) Chunsheng et al.; Licensee Bentham Open.

This is an open access article licensed under the terms of the Creative Commons Attribution Non-Commercial License (http://creativecommons.org/licenses/by-nc/3.0/) which permits unrestricted, non-commercial use, distribution and reproduction in any medium, provided the work is properly cited. 